\title{
Reflections on the Resource Utilization of Livestock and Poultry Breeding in Agricultural Areas
}

\author{
Li Ming Quan \\ Nanchong City Committee Office of the Chinese People's Political Consultative Conference, Nanchong, China \\ Email address: \\ 617738477@qq.com \\ To cite this article: \\ Li Ming Quan. Reflections on the Resource Utilization of Livestock and Poultry Breeding in Agricultural Areas. International Journal of \\ Animal Science and Technology. Vol. 4, No. 4, 2020, pp. 77-80. doi: 10.11648/j.ijast.20200404.12
}

Received: July 9, 2019; Accepted: November 16, 2020; Published: November 23, 2020

\begin{abstract}
Objective: In order to find out the current situation of livestock and poultry breeding waste resource utilization in agricultural areas, improve the methods and measures of livestock and poultry breeding sewage resource utilization. research method: According to the analysis of the current situation, the characteristics of livestock and poultry breeding waste resources were clarified. According to the overall requirements of ecological civilization, the specific measures of livestock and poultry breeding sewage resource utilization were put forward. Conclusion and measures: 1. To establish rules and regulations to standardize the utilization of aquaculture sewage resources; 2 . To improve the facilities and consolidate the foundation of resource utilization; 3. To develop and utilize the aquaculture sewage according to local conditions; 4 . To explore new business forms and establish a new mechanism for the resource utilization of aquaculture sewage; 5 . To strengthen guidance and increase government policy investment.
\end{abstract}

Keywords: Farming Area, Livestock and Poultry Breeding, Dirt, Resource Utilization, Thinking

\section{Introduction}

The resource utilization of livestock and poultry culture pollution is a major policy and a major measure implemented in the current treatment of livestock and poultry breeding in China. In recent years, governments at all levels have done a lot of work in the use of the resources of livestock and poultry breeding, and have achieved some results in some places. However, due to the current situation of livestock and poultry breeding characteristics, the use of animal and poultry breeding pollution resources, facing many difficulties and problems, if we do not face up to the problem, according to local conditions to take measures, the use of animal and poultry breeding in agricultural areas and environmental protection will be difficult to achieve the desired effect [1] To this end, the author of the agricultural area livestock and poultry breeding pollution resource utilization of the status quo of field investigation, to further do a good job in the agricultural area livestock and poultry breeding pollution resource utilization has been considered [2].

\section{Difficulties and Problems}

China's agricultural area of livestock and poultry farming industry, has long been attached to the planting industry, livestock and poultry breeding has been as a farmer's household income sideline, livestock and poultry perched, livestock and poultry farms are poor, its traditional habits of producing dirt is used as fertilizer for the cultivation industry to be converted to clear, there is no intention of resource utilization. [3, 4]. At the same time, the pollution of livestock and poultry breeding industry in agricultural areas has its own characteristics: First, some livestock and poultry farmers' owners of livestock and poultry breeding concept backward, environmental protection and livestock and poultry breeding pollution resource sage awareness difference. All along, people only pay attention to the economic benefits of livestock and poultry breeding, lack of harmless treatment concept. Some livestock and poultry farms have not set up the corresponding pollution prevention and control facilities, some livestock and poultry farms livestock and poultry breeding pollution treatment facilities are not perfect, and some livestock and poultry farms although the construction of 
septic tanks, sedimentation ponds, but the utilization rate of livestock and poultry breeding pollution source is very low; The current livestock and poultry farms in agricultural areas lack of resource-based utilization of treatment facilities, livestock and poultry breeding residues are generally ethedium or accumulation fermentation after simple treatment directly used as farm fertilizer back to the field. This way, the treatment of livestock and poultry culture pollution is limited, treatment time and space are limited, once the amount of livestock and poultry breeding pollution increased, farm fertilizer will become a pollutant, serious pollution to the farmland environment, some places due to excessive application of livestock and poultry manure pollution led to crop harvest, and sometimes even the end of the harvest. Third the form of dirt is complex, classification and treatment difficult. The livestock and poultry breeding in the agricultural areas is scattered, mostly for the breeding of livestock and poultry, the input of forage feed is different, the way of feeding is also different. Therefore, in addition to the resulting dung pollution solid, liquid, gas and other forms, harmful ingredients are not the same, which put forward higher requirements for the resource utilization of livestock and poultry culture pollution; Farming area scattered livestock and poultry breeding livestock, long-term lack of supporting treatment, the collection of dirt, transportation, stacking are difficult, difficult to form a scale, classification collection is not easy to do, not conducive to the use of resources and treatment [5]. The characteristics of livestock and poultry breeding pollution in agricultural areas show that, as a resource and other resources, animal and poultry breeding pollution in agricultural areas is different from other resources has its own unique existence mode, and should also adapt to the methods and measures when carrying out the resource utilization of livestock and poultry breeding in agricultural areas. For thinking about the resource utilization of the current livestock and poultry breeding residues.

\section{Methods and Countermeasures}

\subsection{The Establishment of Regulations to Regulate the Use of Livestock and Poultry Breeding Pollution Resources}

The treatment of livestock and poultry breeding in agricultural areas is a new work of easy to move, people's thinking is complex, the behavior habits of livestock and poultry farmers are difficult to change, the work of many, in the agricultural areas to carry out the use of livestock and poultry breeding pollution resources has more difficulties, although the General Office of the State Council in 2017 issued a "7" On the proposal to accelerate the promotion of the resource utilization of livestock and poultry breeding waste (State Office issued (2017) No. 48, clearly the principle requirements, objectives and measures for the resource utilization of livestock and poultry breeding pollution, but these documents in many places are not widely publicized, poor implementation, livestock and poultry farmers know low, The resource utilization of livestock and poultry culture disagement has not yet become a conscious action of livestock and poultry farmers [6]. Therefore, it is necessary to further regulate the use of animal and poultry breeding pollution resources in agricultural areas through the establishment of rules and regulations. Actively carry out legislative research, the introduction of laws or regulations on the resource utilization of livestock and poultry breeding residues, where there are places to carry out pilot tasks of grass-roots legislation, and where the resource utilization of livestock and poultry culture can be incorporated into the scope of environmental protection legislation according to the actual situation of the local area, and the resource utilization of livestock and poultry breeding in agricultural areas is incorporated into the legislative pilot, in the form of laws and regulations, Standardize the behavior of the resource utilization of livestock and poultry breeding pollution, and promote the development of the resource utilization of livestock and poultry breeding pollution. Governments at all levels should, in accordance with the requirements of the State Council documents and in the light of local realities, formulate and introduce methods and measures for the resource utilization of local livestock and poultry breeding, regulate the behavior of the resource utilization of livestock and poultry breeding, and the competent departments of the national industry should urgently formulate technical norms and technical guidelines for the utilization of livestock and poultry pollution resources and widely publicize them, deeply in the hearts of the people, Form a strong atmosphere of public opinion on the use of animal and poultry pollution resources, and by regulating the behavior of livestock and poultry farmers, the use of animal and poultry breeding resources becomes people's conscious action.

\subsection{Improve the Facilities, Solid Resource Utilization of the Basis}

At present, the resource utilization of livestock and poultry culture in agricultural areas is a new work in the development of animal husbandry [7]. The original design, construction of the enclosure, livestock and poultry breeding pollution resource utilization of facilities and equipment is missing, now to carry out livestock and poultry breeding pollution resource utilization is facing many difficulties. In livestock and poultry-scale livestock and poultry farms, livestock and poultry breeding pollution production is more easy to form a batch scale, can independently establish a treatment system for the resource utilization of pollution, independently carry out the work of resource utilization of livestock and poultry breeding pollution. In the agricultural areas where livestock and poultry are raised separately, due to scattered livestock and poultry breeding, scattered dirt piles, easy to cause pollution to the environment, to collect scattered dirt to implement resource utilization, it is necessary to establish the corresponding facilities and equipment. In areas or livestock breeding communities with livestock breeding habits, it is necessary to be equipped with transport equipment for dry heavy dirt, to carry out a short accumulation of dried livestock and poultry manure in livestock and poultry farms, and then 
transported by means of transport to a special storage point for processing and resource utilization, to solve the problem of the collection of dirt. It is necessary to plan the temporary yard of dry manure scientifically to facilitate mechanized batch processing. To support the construction of household biogas digesters, so that livestock and poultry breeding and biogas closely combined, in the livestock and poultry breeding community, can be set in the planning of the construction of biogas power generation utilization facilities [8]. Strengthen the construction of the pipe network for dirt collection, and after the centralized construction of biogas digesters, the network network can also be used to transmit the generated biogas to farmers or livestock farmers in a timely manner, reducing the waste of biogas production.

\subsection{According to Local Conditions, Classification and Development of Livestock and Poultry Breeding Residues}

The resource utilization of livestock and animal breeding in agricultural areas has been carried out in some places due to the attention of local government [9], but the low utilization rate of the construction of facilities and equipment, poor pollution control, low efficiency, some local construction of facilities and equipment seriously divorced from reality, not only waste of financial and human resources, but also can not reach the effect of the use of livestock and poultry breeding pollution resources, Some also contribute to formalism at work. In the overall control of livestock and poultry breeding pollution adhere to the "source reduction, process control, terminal utilization" of the general principle [10]. In the actual work, the resource utilization of livestock and poultry breeding in agricultural areas should be used according to local conditions, classified and developed, and different types of dirt species should be used for different regions, different breeds and different sizes. Based on the use of fertilizer, as raw materials for organic fertilizer, we should strictly formula production, strengthen the detection of toxic and harmful substances, and strictly prohibit toxic and harmful substances in fertilizer exceeding the standard. Adopt ingesting economical and efficient treatment mode, fertilizer, gas, gas, electricity, to achieve the near use of manure in place, reduce the cost of resource utilization of livestock and poultry breeding. The foul-smelling treatment of livestock and poultry breeding can be used to plant flowers and plants, plant trees, absorb some toxic and harmful substances in the air through the adsorption of plants, build garden-style, garden-style livestock and poultry farms, reduce the discharge of foul odor and harmful gases, purify the air, reduce the pollution of the air from livestock and poultry breeding [11]. The dry manure part produced by livestock and poultry, when the local planting industry can not and use as fertilizer, to collect and stack separately, use the time difference, the production of organic fertilizer, as a commodity, bought to other planting owners as fertilizer, thin part of the feces, urine and contaminated pad grass, can be unified into the biogas digester fermentation, the production of biogas, as fuel. The construction of biogas digester is not the bigger the better, according to the amount of feces of livestock and poultry to determine the size of the construction of biogas digesters, biogas digesters filling is not enough, biogas digesters empty, biogas pressure is not enough, affecting the use of biogas, biogas digesters built small, and can not effectively purify livestock and poultry culture residues [12]. When using livestock and poultry cultured dirt as biogas fillers, livestock and poultry farmers should scientifically make and properly match other draft rod leaves in order to improve their gas production performance and achieve better gas production effect.

\subsection{Explore the New Industry, the Creation of Livestock and Poultry Breeding Pollution Resource Utilization of New Mechanisms}

The resource utilization of livestock and poultry breeding in agricultural areas is a long-term and continuous work, and it is necessary to form a long-term work dynamic mechanism. The resource utilization of livestock and poultry culture diversity should adhere to the diversification of the main body of input, storage and transportation and transformation. Township can combine biogas digester management and biogas clearance, strengthen the clearance and management of livestock and poultry breeding pollution, and form a professional team of professional collection and clearance of livestock and poultry culture [13]. At the same time, the establishment of relevant professional cooperatives and associations, the development of service charters and conventions, responsible for the collection and removal of excess livestock and poultry culture of livestock and poultry farmers. Encourage the use of private funds to invest in the construction of animal and poultry breeding pollution resource utilization facilities, and participate in the development and marketing of new products and other links, the government and give certain subsidies and incentives, the formation of specialized production, market-oriented operation of livestock and poultry breeding pollution treatment mechanism, so that the agricultural area livestock and poultry breeding pollution resources in all aspects of the management, Some people caught, put the agricultural area livestock and poultry breeding pollution resource utilization work into practice [13].

\subsection{Strengthen Guidance, Increase Government Policy Input}

The resource utilization of livestock and poultry breeding in agricultural areas is a new subject in the development of livestock and poultry breeding industry, involving a number of subjects, there are many difficulties and contradictions in the start-up of work, all levels must strengthen guidance, increase policy input, the state should integrate the resource utilization of livestock and poultry breeding in agricultural areas into the national rural revitalization strategy, As an important measure to protect the blue water and blue sky and an important part of the construction of the rural environment, it is very important to put the improvement of facilities and equipment for the resource utilization of livestock and poultry breeding. 
Governments at all levels should incorporate the resource utilization of livestock and poultry breeding into the agenda, study the arrangement together with environmental protection work, strengthen supervision and assessment and reward and punishment, and stimulate the enthusiasm of cadres and masses to carry out the work of utilizing the pollution resources of livestock and poultry breeding [14]. All localities should invest financially to support the construction and allocation of infrastructure and equipment for the resource utilization of livestock and poultry culture, encourage livestock and poultry farmers to take the initiative to carry out the work of resource utilization of livestock and poultry culture, and give appropriate preferential treatment in investment financing and land use.

To carry out the resource utilization of livestock and poultry breeding in agricultural areas is a great good thing for the benefit of the country and the people, and the benefit of the children and grandchildren in the present [15]. Therefore, in the development of modern animal husbandry, we should attach great importance to the resource utilization of livestock and poultry breeding residues, not only to actively cultivate the livestock industry, but also to protect the environment, so that livestock and poultry breeding and environmental protection, to deal with the relationship between the use of livestock and poultry breeding waste and the protection of the environment, to promote the steady upgrading of the livestock industry and structural optimization, So that the rural livestock and poultry industry to obtain better, longer-term development opportunities!

\section{Conclusion and Summary}

In short, in livestock and poultry breeding in agricultural areas, the resource utilization of breeding wastes is one of the important methods to solve the breeding pollution and realize the coordination and unification of breeding and environment. Due to the neglect of environmental protection in traditional livestock and poultry breeding, the sewage treatment methods and measures of breeding are not supporting each other, and environmental pollution incidents often occur. Therefore, in order to protect the environment and ensure the healthy development of the breeding industry, people should pay more attention to the resource utilization of sewage from livestock and poultry breeding, take practical measures, establish rules and regulations, and regulate the resource utilization behavior of sewage from livestock and poultry breeding. Improve facilities, tamp the foundation of resource utilization; According to local conditions, classified development and utilization of aquaculture sewage; Explore new forms of business and create a new mechanism for the reclamation of sewage; We will strengthen guidance and increase input into government policies. In doing so, it can not only promote the sustainable and healthy development of livestock and poultry industry, but also maintain the harmony between human and the environment.

\section{References}

[1] Li Mingquan Thoughts on The Development of Animal Husbandry under the New Normal Economy (J) China's Pig Industry, 2015, 5th issue.

[2] Li Mingquan Thoughts on the Coordinated Development of Breeding and Environment (J) China's Pig Industry, July, 2017.

[3] Li Mingquan's Reform and Innovation of Pig Breeding Model in rural Areas (J) China's Pig Industry, 2013, 5th issue.

[4] Li Mingquan "Five Difficulties" in the Development of Modern Animal Husbandry (J) Chinese Animal Husbandry, 2014. $10^{\text {th }}$.

[5] Li Mingquan Road to The Stable Development of Animal Husbandry in the Whole Agricultural Area (J) China Animal Husbandry, 2012, 8th Issue.

[6] Wang Zhibi Comprehensive Solutions and Applications of Manure Treatment in Farm (J) Livestock and Poultry Industry, Issue 9, 2018.

[7] Li Mingquan, The Way out of aquaculture under the heavy responsibility of Ecological Civilization (J) Sichuan Animal Husbandry and Veterinary science, Vol. 4, 2018.

[8] Han Yongqin, On Epidemic Prevention and Control Technology of Livestock and Poultry in Standardized Farms (J), Modern Animal Husbandry Technology, Vol. 4, 2019.

[9] Gao Yanbing, Liu Jiaying, Li Huawu et al. Analysis of Pollution Prevention and Control Models in Livestock and Poultry breeding (J) Hubei Animal Husbandry and Veterinary Science, 3rd Issue, 2019.

[10] Li Mingquan Brief Analysis of the difficulties and problems faced by the harmless treatment of pig carcasses infected with epidemic Disease (J) China's Pig Industry, 2014, Issue 4.

[11] Zhang Lijun, Zhang Chunliu, Liu Xiaogang, Environmental Pollution caused by Rural Breeding and Countermeasures (J), Livestock and Poultry Industry in China, Vol. 4, 2019.

[12] Guo Xiaodong, Ren Xizhen, Pollution Analysis and Countermeasures of Rural Livestock and Poultry Breeding (J), Resource Conservation and Protection, No. 11, 2018.

[13] Li Mingquan, Improving the construction quality of biogas Project and Strengthening the Pollution Control Function of biogas breeding (J) Chinese Pig Industry, 2019, 1st Issue.

[14] xu Weipu, Chen Guobin, Liu Junliang, et al. Environmental Pollution and Prevention strategies of large-scale farming $(\mathrm{J})$. Environmental Science, S1, 2004.

[15] Ma Chengde Analysis on prevention and Control Measures for Environmental Pollution in Cattle and Sheep Breeding (J), China Animal Husbandry and Veterinary Digest, 1st Issue, 2017. 